Case Report

\title{
Spontaneous Ureterocolic Fistula between Nonfunctioning Kidney Transplant Ureter and Colon in Setting of Diverticulitis
}

\author{
Madison K. Krischak $\mathbb{D}^{1},{ }^{1}$ Jeffrey R. Ord $\mathbb{D}^{1},{ }^{1}$ Ashton A. Connor $\mathbb{D}^{\circ},{ }^{2}$ and Andrew S. Barbas $\mathbb{D}^{2}$ \\ ${ }^{1}$ Duke University School of Medicine, 2608 Erwin Rd, Suite 210, Durham, NC 27705, USA \\ ${ }^{2}$ Division of Abdominal Transplant Surgery, Department of Surgery, Duke University School of Medicine, 2301 Erwin Rd, Durham, \\ NC 27705, USA
}

Correspondence should be addressed to Madison K. Krischak; madison.krischak@duke.edu

Received 8 January 2021; Accepted 6 July 2021; Published 21 July 2021

Academic Editor: Mariano Ferraresso

Copyright (c) 2021 Madison K. Krischak et al. This is an open access article distributed under the Creative Commons Attribution License, which permits unrestricted use, distribution, and reproduction in any medium, provided the original work is properly cited.

Ureterocolic fistula is a rare condition that most commonly occurs in the setting of diverticular disease. The development of a ureterocolic fistula following kidney transplantation is even rarer, with no prior cases in the literature to our knowledge. We describe the case of a patient with three prior failed kidney transplants who developed a fistula between the sigmoid colon and nonfunctioning renal transplant ureter in the setting of diverticulitis.

\section{Introduction}

The formation of fistulas in the setting of a diverticular disease is relatively rare, occurring in only $1 \%$ of cases [1]. Fistula formation with the ureter is particularly rare, with only 11 previous cases reported in the literature [2]. Ureterocolic fistulas most commonly affect the left ureter given its proximity to the sigmoid colon. Presentation, diagnosis, and management of this condition vary according to the functional status of the affected upper urinary tract. In patients with functional kidneys, the recommended treatment is resection of the affected colon without surgical intervention of the urinary system. However, in the setting of a nonfunctioning kidney, resection of the affected urinary system is indicated [3]. We present a case of ureterocolic fistula in the setting of nonfunctional renal transplant graft with subsequent colonic resection and transplant ureteronephrectomy. To our knowledge, this is the first published example of a ureterocolic fistula with a nonfunctional renal transplant ureter in the setting of diverticulitis.

\section{Case Presentation}

The patient is a 57 -year-old female with type 1 diabetes complicated by end-stage renal disease. The patient had an extensive transplant history including a kidney transplant in 1996, followed by a simultaneous kidney and pancreas transplant in 2000, followed by another kidney transplant in 2009. All three kidneys failed due to chronic rejection. The patient had returned to hemodialysis and was anuric at baseline. Her pancreatic graft remained functional, and she was continued on immunosuppression with $0.5 \mathrm{mg}$ tacrolimus and $5 \mathrm{mg}$ prednisone daily. She underwent transplant nephrectomy of the most superficial nonfunctional renal graft in 2017 due to its enlarging size and discomfort. The patient's other significant medical history included hepatitis C, history of pneumocystis pneumonia, chronic anemia, severe peripheral artery disease, and left lower extremity nonhealing wound status post amputation.

The patient presented to our institution's emergency department with abdominal pain and low-grade fevers for 48 hours. At presentation, her vital signs were stable and she was nontoxic appearing with localized tenderness in the left lower quadrant. Initial computed tomography (CT) imaging of the abdomen and pelvis demonstrated a fistulous connection between the sigmoid colon and the patient's left nonfunctioning kidney transplant ureter (Figures 1 and 2). The patient was started on intravenous piperacillintazobactam for broad antibiotic coverage. Flexible sigmoidoscopy and biopsy were completed and were negative for evidence of malignancy as a cause of fistula. She was taken 


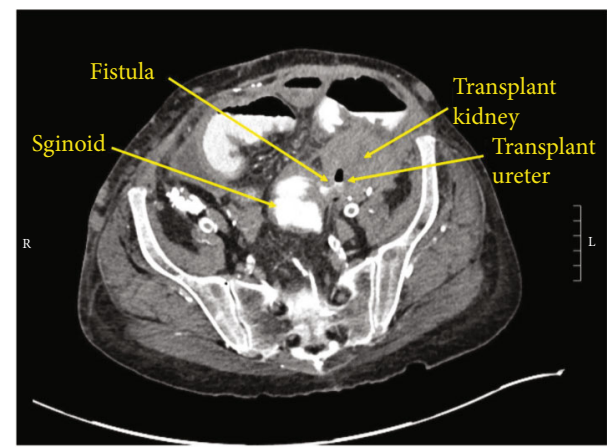

Figure 1: Axial CT of the abdomen and pelvis with rectal contrast demonstrating fistulous connection between the sigmoid colon and left renal transplant ureter.

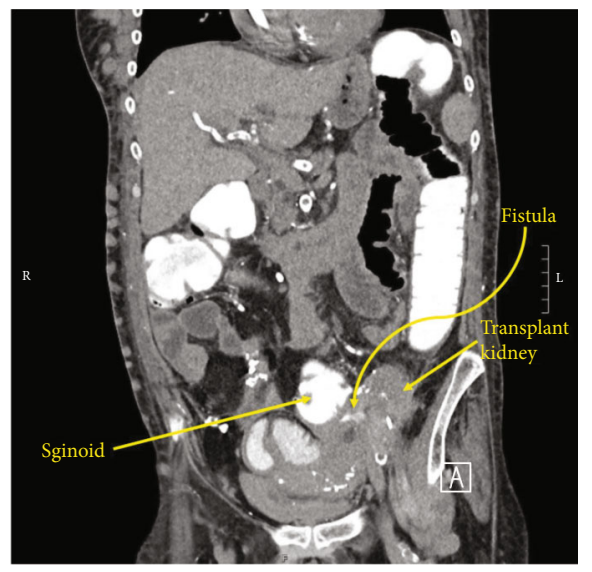

FIGURE 2: Sagittal CT of the abdomen and pelvis with rectal contrast demonstrating fistulous connection between the sigmoid colon and left renal transplant ureter.

to the operating room for sigmoid colectomy with colostomy and transplant ureteronephrectomy.

In the operating room, the fistula was identified. The affected colon was mobilized, proximally and distally, and removed. Following colonic resection, the transplant ureter was identified, ligated, and divided near the bladder. The kidney graft was mobilized and removed. Hemostasis was achieved in the pelvis, but a laceration of the spleen was identified, and splenectomy was required.

Once hemostasis was achieved, the colorectal team formed an end colostomy. At this point in the case, the patient had significant hypotension requiring placement of a central line, increasing vasopressor support, and blood products. At the end of the case, she was transported to the intensive care unit (ICU) hemodynamically unstable and intubated. Operating room pathology demonstrated diverticulosis with perforation and explanted kidney with extensive necrosis and pyonephrosis. Given intraoperative findings, blood cultures were obtained and the patient was continued on piperacillintazobactam with addition of vancomycin and micafungin at recommendation of infectious disease colleagues.

Postoperatively, the patient required vasopressor support and ICU level care. She returned to hemodialysis on post-op day (POD) 1 and was weaned off vasopressors on POD 2. Blood cultures remained negative at 48 hours; thus, antimicrobials and antifungals were discontinued. The patient's recovery was otherwise uneventful. She was deemed medically fit for discharge on POD 10.

Two weeks following discharge, the patient represented in septic shock and was found to have diffuse purulent peritonitis upon exploratory laparotomy. Her subsequent hospital stay was prolonged and complicated, although she was eventually discharged to a skilled nursing facility. Unfortunately, she passed away shortly thereafter during a subsequent admission for osteomyelitis.

\section{Discussion}

Fistula formation in the setting of diverticulitis is rare, occurring in only $1 \%$ of cases [1]. The most common fistulas formed are colovesical and colovaginal [4], with ureterocolic fistulas being extremely rare in the literature. Historically, ureterocolic fistulas have formed in the setting of tuberculosis. Other notable causes include ureterolithiasis, pyelonephritis, prior surgical intervention, trauma, Crohn's disease, diverticulitis, radiation therapy, and malignancy [5]. Ureterocolic fistula due to diverticulitis is exceedingly rare, with only 11 prior cases reported in the literature to our knowledge [2]. Of those cases, none have taken place in patients with nonfunctioning kidney transplant ureters.

Diagnosis of ureterocolic fistula is dependent on the functional status of the affected urinary system. Importantly, most ureterocolic fistulas will present with both urinary symptoms such as urinary tract infection, pneumaturia, and fecaluria, and abdominal pain $[2,6]$; however, in the setting of a nonfunctional transplant ureter and kidney, urinary symptoms are less likely. Thus, awareness of this complication in absence of characteristic symptoms is key when considering the differential for abdominal pain in this population. Diagnosis is typically made via retrograde urethrography, excretory urography (showing contrast material entering the bowel), or barium enema (with contrast media entering the ureter) [5]. However, in the setting of a nonfunctional transplant kidney these tests are of limited value and diagnosis more heavily relies on CT imaging. When a ureterocolic fistula is suspected, appropriate imaging studies are essential for quick diagnosis and prevention of urosepsis.

Similar to diagnosis, surgical management of ureterocolic fistula is also dependent on the functional status of the kidney. In cases of functional kidneys, it is recommended that the affected bowel be resected without intervention on the kidney or ureter $[3,5]$. In more severe cases, however, the decision may be made to dissect the fistula and reanastomose the ureter [5]. Some rare cases of conservative management with antibiotics have been reported [7], but this is generally not recommended over surgical management. In the setting of nonfunctioning kidney as in this case, the recommended management includes both resection of the affected bowel and ureteronephrectomy to decrease risk for urosepsis in the nonfunctioning upper urinary tract $[6,8]$.

In this case, surgical management of the patient's ureterocolic fistula was successful, given the patient stabilized and 
achieved adequate ostomy output, ambulation, and pain control with oral medications prior to discharge home. Unfortunately, the patient represented in septic shock two weeks later; however, her presentation is thought to be predominantly a consequence of compromised immune function given her immunosuppressive medications and recent splenectomy. Importantly, asplenic patients are at heightened risk of overwhelming postsplenectomy infection (OPSI) [9]. Although this patient received appropriate antibiotics during her immediate postoperative period, some recommendations suggest that asplenic individuals with particularly high risk of infection, including those with solid organ transplants, should receive daily antibiotic prophylaxis for the duration of their immunosuppression in addition to appropriate vaccines; however, the data is limited and these recommendations vary [10]. While this patient's surgical management of ureterocolic fistula was successful, her postoperative course was significantly impacted by her heightened immunocompromised state and should be a consideration in all asplenic patients with surgically managed ureterocolic fistula.

Discussion of ureterocolic fistula management in the renal transplant population is increasingly important. If caught early in a patient with a functional graft, the graft may be spared and urosepsis avoided. However, early detection of this phenomenon can be difficult in the anuric patient with failed transplant grafts due to lack of symptoms and thus relies heavily on imaging. As the population with renal transplants expands and ages, they face increased risk of diverticular disease. As such, it can be postulated that although rare, the incidence of ureterocolic fistulas in the transplant population may increase over time. Awareness of this complication and application of the appropriate diagnostic tools and surgical management based on the functional status of the renal transplant are crucial.

\section{Data Availability}

No data were used to support this study.

\section{Conflicts of Interest}

The authors declare no conflict of interest.

\section{Authors' Contributions}

Madison K. Krischak participated in writing the work and participated in revising the work critically for important intellectual content (email: madison.krischak@duke.edu). Jeffrey R. Ord participated in revising the work critically for important intellectual content (email: jeffrey.ord@duke.edu). Ashton A. Connor participated in revising the work critically for important intellectual content (email: ashton.connor@duke.edu). Andrew S. Barbas participated in revising the work critically for important intellectual content (email: andrew.barbas@duke.edu).

\section{References}

[1] D. J. Humes and J. West, "Role of acute diverticulitis in the development of complicated colonic diverticular disease and 1 -year mortality after diagnosis in the UK: population-based cohort study," Gut, vol. 1, pp. 95-100, 2012.

[2] M. Q. Almerie, A. Culverwell, J. Gill, and J. D. Harrison, "Spontaneous right-sided ureterocolic fistula: a rare complication of colonic diverticular disease," BMJ Case Reports, vol. 2015, 2015.

[3] W. C. Cirocco, S. R. Priolo, and R. W. Golub, "Spontaneous ureterocolic fistula: a rare complication of colonic diverticular disease," The American Surgeon, vol. 60, no. 11, pp. 832-835, 1994.

[4] J. W. Husted, "An unusual case of pyelonephritis: nephrocolic fistula owing to a ruptured colonic diverticulum," The Journal of urology, vol. 6, pp. 724-726, 1974.

[5] Y. Maeda, S. Nakashima, and T. Misaki, "Ureterocolic fistula secondary to colonic diverticulitis," International Journal of Urology, vol. 5, no. 6, pp. 610-612, 1998.

[6] A. Lao and D. Bach, "Colonic complications in renal transplant recipients," Diseases of the Colon and Rectum, vol. 31, no. 2, pp. 130-133, 1988.

[7] Y. Iwamoto and M. Kato, "A case with fistula formation between a perinephric retroperitoneal abscess, a ureter and a descending colon: successful outcome after conservative management," Canadian Urological Association Journal, vol. 8, no. 9-10, pp. E644-E646, 2014.

[8] A. Pai, J. Coralic, J. J. Park, S. Marecik, and L. M. Prasad, "Spontaneous ureterocolic fistula secondary to diverticulitis: report of a case and review of literature," International Journal of Colorectal Disease, vol. 29, no. 3, pp. 417-418, 2014.

[9] P. E. Schwartz, S. Sterioff, P. Mucha, L. J. Melton 3rd, and K. P. Offord, "Postsplenectomy sepsis and mortality in adults," Jama, vol. 248, no. 18, pp. 2279-2283, 1982.

[10] C. Kaplinsky and Z. Spirer, "Post-splenectomy antibiotic prophylaxis-unfinished story: to treat or not to treat?," Pediatric blood \& cancer, vol. 47, no. S5, pp. 740-741, 2006. 